\title{
Elaboração e validação da cartilha "Higiene do Sono para Crianças"
}

Development and validation of the "Sleep Hygiene for Children" booklet

Elaboración y validación de la cartilla "Higiene del sueño para niños"

Nathalie Sales Llaguno ${ }^{1}$ @ h ntps://orididorg//0000-0002-3416-6178

Eliana Moreira Pinheiro' ${ }^{1}$ ic https://orcid.org/0000-0003-1108-1080

Ariane Ferreira Machado Avelar' ${ }^{1}$ ib https://orcid.org/0000-0001-7479-8121

Como citar:

Llaguno NS, Pinheiro EM, Avelar AF. Elaboração e validação da cartiliha "Higiene do Sono para Crianças". Acta Paul Enferm. 2021;34:eAPE001125.

DOI

http://dx.doi.org/10.37689/actaape/2021A0001125

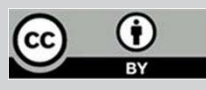

Descritores

Higiene do sono; Sono; Criança; Desenvolvimento infantil; Educação em saúde

Keywords

Sleep hygiene; Sleep; Child; Child development; Health education

Descriptores

Higiene del sueño; Sueño; Ninõ; Desarrollo infantil; Educación en salud

\section{Submetido}

15 de Maio de 2020

Aceito

7 de Dezembro de 2020

\section{Autor correspondente}

Nathalie Sales Llaguno

E-mail: nathalie.llaguno@gmail.com

\section{Resumo}

Objetivo: Elaborar e validar cartilha sobre higiene do sono para crianças escolares.

Métodos: Estudo de abordagem metodológica, realizado no período de setembro de 2018 a outubro de 2019, seguindo as etapas de levantamento bibliográfico, elaboração e validação de conteúdo da cartilha por especialistas. 0 instrumento para validação, encaminhado a três profissionais da área da saúde, foi composto por sete itens, divididos em subitens, com respostas dispostas em escala tipo Likert. Os dados obtidos foram tabulados em planilha do Excel. Considerou-se adequado os valores da proporção de concordância dos subitens entre os examinadores (índice de validade do conteúdo) acima de $80 \%$.

Resultados: A cartilha foi validada após a segunda rodada de avaliação, atingindo índice de validade de 85,7\% do conteúdo total e sendo composta por quatro temas: "0 que é higiene do sono?", "Importância do sono", "Como realizar a higiene do sono?" e "Guia rápido de recomendações de higiene do sono".

Conclusão: A cartilha de higiene do sono para crianças, validada por especialistas na segunda rodada, poderá auxiliar a criança a entender a importância do sono para seu desenvolvimento e obter hábitos adequados de sono e de autocuidado.

\section{Abstract}

Objective: To develop and validate a booklet on sleep hygiene for school children.

Methods: Methodological study conducted from September 2018 to October 2019, following the steps of bibliographic survey, development and content validation of the booklet by experts. The instrument for validation, sent to three health professionals, was composed of seven items, divided into sub-items with responses arranged on a Likert-type scale. Data obtained were tabulated in an Excel spreadsheet. Values of the proportion of agreement of sub-items between experts (content validity index) above $80 \%$ were considered appropriate.

Results: The booklet was validated after the second evaluation round, reaching a validity rate of $85.7 \%$ of the total content. It comprised the following four themes: "What is sleep hygiene?", "Importance of sleep", "How to practice sleep hygiene?" and "Sleep hygiene recommendations quick guide".

Conclusion: The sleep hygiene booklet for children, validated by experts in the second round, can help children understand the importance of sleep for their development and obtain appropriate sleep and self-care habits.

\section{Resumen}

Objetivo: Elaborar y validar una cartilla sobre higiene del sueño para niños escolares. 
Métodos: Estudio de enfoque metodológico, realizado en el período de septiembre de 2018 a octubre de 2019, que siguió las etapas de recopilación bibliográfica, elaboración y validación del contenido de la cartilla por especialistas. El instrumento para la validación, que fue enviado a tres profesionales del área de la salud, estaba compuesto por siete ítems, divididos en subítems, con respuestas dispuestas en escala tipo Likert. Los datos obtenidos fueron tabulados en planilla de Excel. Los valores de la proporción de concordancia de los subítems entre los examinadores (índice de validez de contenido) superiores a $80 \%$ se consideraron adecuados.

Resultados: La cartilla fue validada después de la segunda ronda de evaluación, con un índice de validez del contenido total del 85,7 \%. El material está compuesto por cuatro temas: “¿Qué es la higiene del sueño?”, "Importancia del sueño”, “¿Cómo realizar la higiene del sueño?” y “Guía rápida de recomendaciones de higiene del sueño".

Conclusión: La cartilla de higiene del sueño para niños, validada por especialistas en la segunda ronda, podrá ayudar a los niños a entender la importancia del sueño para el desarrollo y a obtener hábitos adecuados de sueño y de autocuidado.

\section{Introdução}

O sono da criança é extremamente importante para o desenvolvimento saudável, pois está relacionado ao crescimento, à aprendizagem e ao funcionamento do organismo. ${ }^{(1,2)}$ Quando a criança não dorme adequadamente pode haver prejuízo no desempenho escolar, problemas emocionais e comportamentais, diminuição da função cognitiva e outros distúrbios de saúde que podem resultar em menor qualidade de vida e bem-estar, além do risco de desenvolver doenças psiquiátricas na adolescência ou na idade adulta. ${ }^{(3,4)}$

O tempo e a qualidade do sono dependem de fatores genéticos, fisiológicos, ambientais e dos hábitos de sono, definidos como comportamentos culturalmente aprendidos e adotados pelo indivíduo ou cuidador. ${ }^{(2,5)}$

No resultado preliminar de um estudo conduzido com crianças em idade escolar no domicílio, que teve entre os objetivos mensurar o tempo total de sono com uso do actímetro por 15 noites consecutivas, identificou-se que as crianças dormiam cerca de oito horas por dia, ou seja, menos que as 9 horas diárias recomendadas pela literatura para esta faixa etária. ${ }^{(6,7)}$ Atualmente, nota-se certa dificuldade em proporcionar um sono adequado para as crianças devido a hábitos como uso demasiado de eletrônicos e o horário estabelecido para o início das aulas pela manhã. ${ }^{(8,9)}$

Com o objetivo de desenvolver hábitos saudáveis para a promoção do sono das crianças, a literatura recomenda o emprego da higiene do sono, que caracteriza-se como um conjunto de recomendações que visam mudança no comportamento, nas atividades diárias e no ambiente. ${ }^{(10)}$ Estudos que avalia- ram o sono de crianças mostraram resultados mais favoráveis quanto à qualidade e quantidade após a implementação de determinadas intervenções. ${ }^{(11,12)}$

A conscientização da importância da promoção da higiene do sono pode ocorrer a partir da educação em saúde, que prepara o indivíduo para o autocuidado e reduçáo de danos, sendo realizada pelo profissional de saúde, em qualquer local de atuação, como escolas, unidades básicas de saúde ou hospitais. Nesse contexto, o enfermeiro na escola também desempenha papel fundamental nas açóes de saúde com função de prevenção, manutenção e restabelecimento da saúde. ${ }^{(13)}$

De maneira geral, uma alternativa muito utilizada na educação em saúde é o emprego de materiais impressos educativos que facilitam a compreensão e aumentam a adesão ao tema, favorecendo a adoção de hábitos mais saudáveis, principalmente tratando-se da população infantil. ${ }^{(14)}$ Assim, considerando-se a importância do sono para o desenvolvimento infantil e a da confecção de material educativo direcionado às crianças, esse estudo tem por objetivo elaborar e validar uma cartilha sobre higiene do sono para crianças escolares, a fim de contribuir para aquisição de hábitos mais adequados para a promoção do sono infantil.

\section{Métodos}

Estudo de abordagem metodológica, com foco no desenvolvimento e avaliação de uma tecnologia, realizado no período de setembro de 2018 a outubro de 2019, desenvolvido nas seguintes etapas: levantamento bibliográfico, elaboração e validação de conteúdo da cartilha por especialistas. ${ }^{(15)}$ 


\section{Levantamento bibliográfico}

A cartilha foi elaborada a partir de levantamento bibliográfico realizado entre setembro de 2018 a maio de 2019, nas bases Scielo e Pubmed, utilizando-se Descritores em Ciências da Saúde/Medical Subject Headings (DeSC/MeSH): "Materiais de Ensino", "Higiene do Sono" e "Serviço de Saúde Escolar" em inglês e português, associados ou náo por meio do operador booleano $A N D$, delimitando-se o período de publicação de 2010 a 2018. Destaca-se que dada a escassez de materiais desenvolvidos para uso em escolas e a fim de ampliar o levantamento, buscou-se conteúdos sobre a temática em sites eletrônicos de hospitais, centros clínicos gerais e ou infantis, além de associaçóes de estudos do sono dos Estados Unidos da América, Austrália, Portugal, Inglaterra e Brasil. ${ }^{(3,16,17)}$

\section{Elaboração}

A cartilha foi confeccionada no programa Microsoft Office Powerpoint ${ }^{\oplus}$, versão 2019 (16.0.12827.20200) 32 bits, contendo as informaçôes obtidas a partir do conteúdo identificado e figuras elaboradas pela pesquisadora principal, customizadas por designer de jogos digitais no aplicativo PicsArt Editor de Foto e Vídeo versão 15.0 (aplicativo gratuito e disponível para sistema operacional Android e IOS), a fim de tornar o material mais atrativo para o público-alvo.

\section{Validação de conteúdo}

Após a elaboração da cartilha foram selecionados avaliadores por meio de busca por "Assunto" na Plataforma Lattes (www.lattes.cnpq.br) com o termo "sono", nas bases de doutores e demais pesquisadores. Elencou-se aleatoriamente cinco profissionais que foram contatados por e-mail convite, que continha a cartilha proposta, o termo de consentimento livre e esclarecido e o instrumento para validação do conteúdo. Ressalta-se que o número ímpar de experts foi considerado para evitar empate nas opinióes durante o processo de validação.

Obteve-se retorno de três profissionais, sendo uma enfermeira, uma biomédica e uma fisioterapeuta, todas com publicaçóes sobre a temática, títulos de mestre e/ou doutor.
Para avaliação do conteúdo da cartilha, elaborou-se um instrumento de coleta de dados ${ }^{(18)}$ organizado em 7 itens divididos em subitens, para que o avaliador selecionasse uma única resposta em escala tipo Likert, de acordo com sua opiniáo sobre o subitem: DF - Discordo Fortemente, D - Discordo, C - Concordo, CF - Concordo Fortemente e NS - Náo Sei. Além disso, após cada item havia um campo designado para "Sugestóes".

A fim de atingir o objetivo de validação do conteúdo da cartilha, o instrumento foi composto por: 1 . Conteúdo (1.1 O conteúdo está correto cientificamente.; $1.2 \mathrm{O}$ conteúdo está apropriado ao público-alvo.; $1.3 \mathrm{O}$ conteúdo é suficiente para atender às necessidades do público-alvo.; $1.4 \mathrm{~A}$ sequência do texto é lógica.; $1.5 \mathrm{~A}$ apresentação do conteúdo favorece a aprendizagem da temática.); 2 . Linguagem (2.1 O estilo da redação é compatível com o público-alvo (crianças de 6 a 10 anos).; 2.2. A escrita utilizada é atrativa.; 2.3 A linguagem do texto é clara e objetiva.); 3. Ilustraçóes (3.1 As ilustrações são pertinentes ao conteúdo do material e elucidam o conteúdo.; 3.2 As ilustrações são claras e transmitem facilidade de compreensão.; 3.3 As ilustraçôes possuem qualidade gráfica.; $3.4 \mathrm{~A}$ quantidade de ilustraçôes está adequada para o conteúdo do material educativo.; $3.5 \mathrm{~A}$ presença de cada uma das figuras na cartilha é relevante.); 4. Layout (4.1 O tipo de letra utilizada facilita a leitura.; 4.2 A composição visual está atrativa e bem organizada.; 4.3 O formato (tamanho) do material educativo e o número de páginas estão adequados a faixa etária.; 4.4 A disposição do texto está adequada.); 5 . Motivação (5.1 O conteúdo está motivador e incentiva prosseguir a leitura.; $5.2 \mathrm{O}$ conteúdo poderá despertar o interesse da criança leitora.; $5.3 \mathrm{O}$ conteúdo poderá atender às dúvidas., esclarecer e educar a criança quanto a higiene do sono.); 6. Cultura (6.1 O texto está compatível com o público-alvo, atendendo aos diferentes perfis dos usuários.; 6.2 A cartilha é indicada para uso como recurso educativo na higiene do sono.); e 7. Aplicabilidade (7.1 A cartilha possui aplicabilidade prática para uso com crianças de 6 a 10 anos.).

Os resultados foram tabulados em planilha Microsoft Office Excel $^{\oplus}$, versão 2019 
(16.0.12827.20200) 32 bits, sendo apresentados por meio de estatística descritiva. A proporção de concordância entre as respostas dos examinadores para cada subitem do instrumento de avaliação foi calculada pelo índice de validade do conteúdo (IVC), expresso pela razão entre o número de subitens pontuados como "concordo fortemente" ou "concordo" e o número total de avaliaçóes realizadas. O IVC foi verificado para cada item do instrumento (IVC parcial) e para o instrumento como um todo (IVC total), sendo considerados adequados os valores acima de $80 \%$ e o conteúdo validado segundo o IVC total igual ou superior a $80 \% .^{(19)}$

Foram realizados os procedimentos éticos-legais para início da pesquisa, sendo aprovada pelo Comitê de Ética em Pesquisa da Universidade Federal de São Paulo, sob número 0370/2018.

\section{Resultados}

A cartilha foi elaborada a partir da leitura na íntegra de 23 artigos e 6 publicaçóes de associaçóes do sono, sendo composta pelas recomendaçóes mais citadas e adequadas de higiene do sono para a faixa etária escolar. Para validação do conteúdo participaram três juízes na primeira rodada, dois com título de mestrado e um com título de doutorado, que avaliaram a primeira versáo da cartilha por meio do questionário de validação. A cartilha em sua primeira versão apresentava-se dividida em: "Páginas 1 a 4 - Introdução à higiene do sono", "Páginas 5 a 7 - Importância do sono", "Página 8-Quando devemos dormir?" e "Páginas 9 a 14 - Como realizar a higiene do sono?". Obteve-se IVC total de 58,4\%, portanto, abaixo do valor estipulado para validação. A figura 1 apresenta o IVC parcial para cada item do instrumento e o IVC total na primeira rodada de avaliação.

As sugestóes dos especialistas abrangeram principalmente o item "Linguagem", com indicação para substituição de termos técnicos por palavras mais usuais, para que fosse mais compreensível para as crianças. Sugeriu-se também a inclusão de uma lista que favorecesse o acesso rápido das crianças às recomendaçóes para higiene do sono.

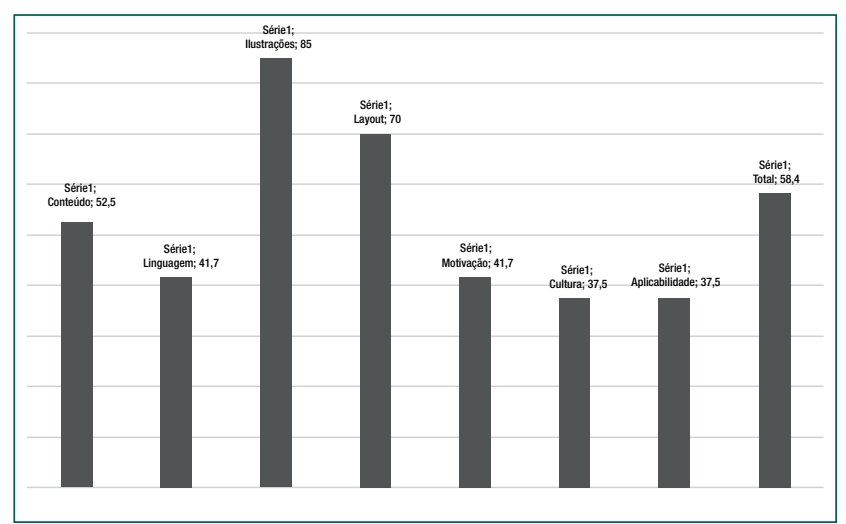

Figura 1. Índice de validade de conteúdo (IVC) parcial e total na primeira rodada

Assim, acatadas as sugestóes dos especialistas, os temas foram reorganizados, a apresentação inicial do conteúdo ficou mais sucinta, os termos utilizados para as recomendaçóes de higiene do sono foram simplificados e melhor elaborados para compreensão do público-alvo, e por fim, incluiu-se o guia rápido de recomendaçóes.

A segunda versão da cartilha enviada aos mesmos três juízes apresentava-se dividida em: "Página 1- O que é higiene do sono?", "Páginas 2 a 3 - Por que cuidar do sono é tão importante?", "Páginas 4 a 9 - Como podemos praticar a higiene do sono no dia-a-dia?" e "Página 10 - Relembrando o principal...". A figura 2, apresenta o IVC parcial para cada item do instrumento e o IVC total $(85,7 \%)$ na segunda rodada de avaliação pelos juízes. $\mathrm{O}$ item "Linguagem" não atingiu a porcentagem mínima devido ao subitem 2.2 A escrita utilizada é atrativa, entretanto, a avaliação dos outros itens atingiu o IVC total acima do estipulado.

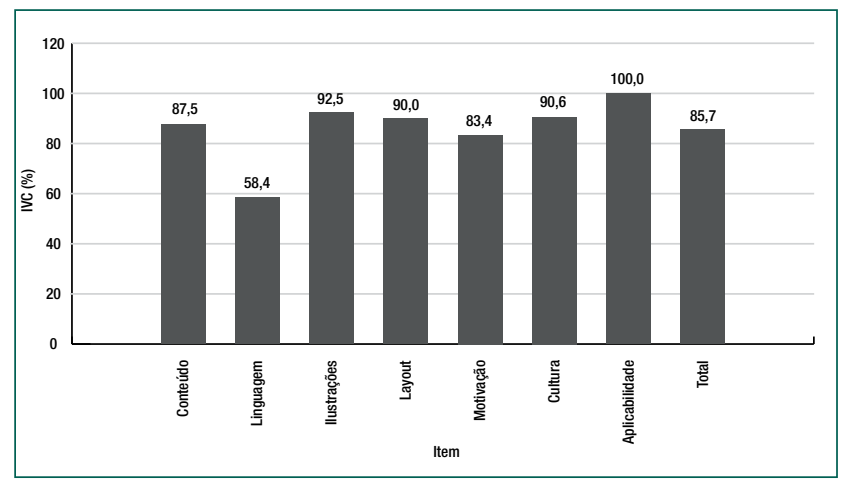

Figura 2. Índice de validade de conteúdo (IVC) parcial e total na segunda rodada 


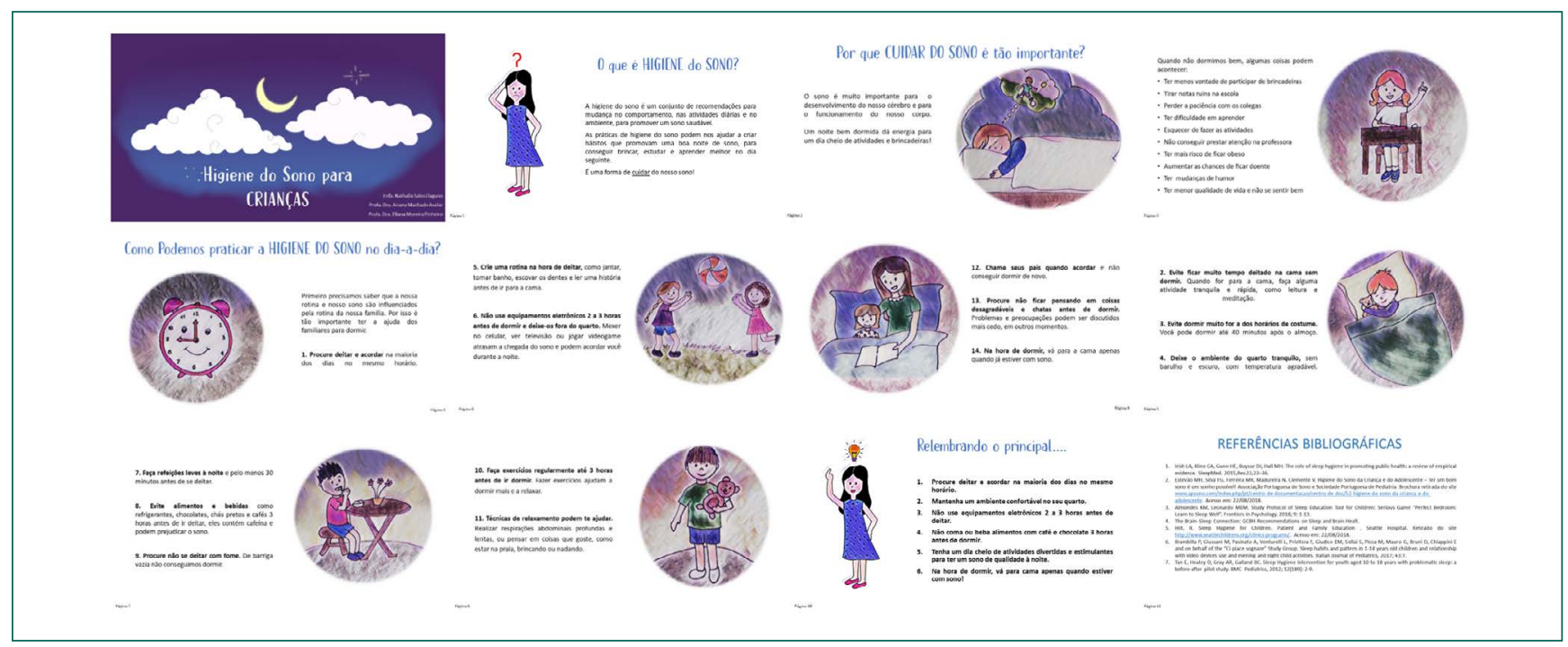

Figura 3. Versão Final da Cartilha "Higiene do Sono para Crianças"

O conteúdo da cartilha foi separado em temas por páginas, dispostos em parágrafos ou em tópicos com palavras simples, incluindo apenas as informações necessárias para compreensão da criança. Inicialmente, com enfoque no entendimento da criança sobre a importância do sono e na sequência, medidas para promoção de um sono adequado.

As ilustrações foram concebidas para relacionar-se com a escrita de cada página e chamar a atenção das crianças com um design colorido. Utilizaram-se as fontes Erstwhile DEMO para títulos com tamanho 50 a 60 e Confortaa para textos com tamanho 20.

A cartilha, denominada "Higiene do Sono para Crianças”, foi composta por capa, 11 páginas ilustradas e quatro temas (Figura 3).

\section{Discussão}

No processo de elaboração e validação de conteúdo da cartilha que orienta hábitos saudáveis para promoção do sono de crianças escolares, evidenciou-se a escassez de estudos que apresentam recomendaçóes robustas à faixa etária escolar, além da não avaliação da cartilha por crianças escolares e do pequeno número de juízes que avaliaram o conteúdo do material, embora tivessem ampla experiência na temática.

A educação em saúde quando inserida no ambiente da escola pelo enfermeiro pode ser uma ferra- menta na promoção à saúde, possibilitando a inserção de hábitos saudáveis e qualidade de vida. ${ }^{(20)}$ Materiais impressos, em formato de cartilhas, folders, panfletos ou livretos, são usados na educação em saúde com o objetivo de divulgar informaçóes consideradas importantes para a prevenção ou tratamento de enfermidades, e possibilitam a interação entre profissionais e a população. ${ }^{(21)}$ É indispensável que o material seja escrito em linguagem acessível, que seja atrativo, objetivo, sucinto, mas que dê orientação significativa sobre o tema, sendo importante ilustrar as orientações para descontrair, incentivar a leitura, torná-lo mais atraente e facilitar o entendimento. ${ }^{(21,22)}$

Dessa forma, a cartilha com ilustrações é uma forma acessível de orientar as crianças sobre a importância do sono e que a adoção de medidas poderá auxiliá-las a obter um sono adequado. Sendo a criança na fase escolar o público-alvo do material, a apresentação da cartilha com características específicas ganha ainda maior importância, pois para que desperte o interesse do leitor e influencie seus hábitos de maneira positiva, deve trazer o lúdico, uma experiência divertida e atrativa. ${ }^{(23,24)}$

Para validação do conteúdo da cartilha, a literatura descreve a necessidade de consideraçóes de especialistas no tema, afinal, além de se obter uma opinião multiprofissional precisa, as sugestôes refinam o material e o tornam oficial. ${ }^{(25)}$ Autores que elaboraram e validaram conteúdo de materiais educativos utilizaram o índice de validade de conteúdo (IVC) para de- 
terminar o consenso entre os especialistas, adotando índice de concordância igual ou superior a 75\%. . $^{(6,27)}$

Após obtenção do IVC total de $85,7 \%$, partiu-se para a avaliação das consideraçóes e sugestôes dos especialistas que foram acatadas na totalidade para melhora da linguagem da versão final da cartilha. É importante frisar que por meio das sugestóes, essa cartilha foi desenvolvida observando as diferenças individuais das crianças de acordo com os cronótipos, que predispóem a uma preferência de horários para dormir e acordar, as fases de maior disposição física e cognitiva, respeitando a percepção individual dos ritmos circadianos e os sincronizadores externos em seres humanos. ${ }^{(28)}$

Nota-se a escassez de materiais educativos na literatura sobre higiene do sono voltados especificamente para as crianças. Os materiais disponíveis em universidades, hospitais e clínicas têm como público-alvo os adultos responsáveis pela criança e enfatizam aspectos referentes ao sono em diferentes faixas etárias, com abordagem de doenças associadas, criação de hábitos e rotinas saudáveis, bem como o preparo do ambiente para o sono. ${ }^{(29-31)}$

Assim, a presente cartilha pretende oferecer à criança o entendimento do que se trata a higiene do sono e porque cuidar do sono é táo importante, sem necessitar da ajuda de um adulto, constituindo-se uma das bases da educação em saúde, a de formar indivíduos com conhecimento, motivação e competência para entender, avaliar e aplicar as informaçôes em saúde, fortalecendo seu autocuidado e tornando-os capazes de transformar a sua realidade. ${ }^{32,33)}$

A participação da família na educação da criança é fundamental, mas enfatiza-se a necessidade de sua participação ativa no processo de aprendizagem para começar a introjetar a importância de suas açóes no contexto do autocuidado em todas as fases do ciclo vital, de modo a realizar de forma autônoma determinadas rotinas tornando-as hábitos para uma vida saudável.

\section{Conclusão}

A cartilha para higiene do sono desenvolvida a partir da identificação de maus hábitos na promoção do sono de crianças escolares foi elaborada e validada por especialistas na segunda rodada. Ela é composta por quatro temas e um guia de recomendaçóes para adoção de hábitos saudáveis para promoção do sono, e poderá auxiliar a criança a obter hábitos adequados de sono e o autocuidado, entendendo a importância do sono para seu desenvolvimento. Dentre as implicações para a prática, enfatiza-se que a cartilha desenvolvida visa dar suporte às açóes de cuidado da enfermeira no ambiente escolar, podendo favorecer a autonomia da criança para o cuidado com o sono, além de possibilitar a atuação como multiplicadora deste conhecimento em sua família.

\section{Colaborações}

Llaguno NS, Pinheiro EM e Avelar AFM contribuíram com a concepção do projeto, análise e interpretação dos dados, redação do artigo, revisão crítica relevante do conteúdo intelectual e aprovação final da versão a ser publicada.

\section{Referências}

1. Bonuck KA, Schwartz B, Schechter C. Sleep health literacy in head start families and staff: exploratory study of knowledge, motivation, and competencies to promote healthy sleep. Sleep Health. 2016;2(1):19-24.

2. Buxton OM, Chang AM, Spilsbury JC, Bos T, Emsellem H, Knutson KL. Sleep in the modern family: protective family routines for child and adolescent sleep. Sleep Health. 2015;1(1):15-27.

3. de Almondes KM, Leonardo ME. Study Protocol of Sleep Education Tool for Children: Serious Game "Perfect Bedroom: Learn to Sleep Well". Front Psychol. 2018;9:1016.

4. Reynaud E, Vecchierini MF, Heude B, Charles MA, Plancoulaine S. Sleep and its relation to cognition and behaviour in preschool-aged children of the general population: a systematic review. J Sleep Res. 2018;27(3):e12636.

5. Chaput JP, Dutil C, Sampasa-Kanyinga H. Sleeping hours: what is the ideal number and how does age impact this? Nat Sci Sleep. 2018;10:421-30.

6. Paruthi S, Brooks LJ, D’Ambrosio C, Hall WA, Kotagal S, Lloyd RM, et al. Consensus statement of the American Academy of Sleep Medicine on the recommended amount of sleep for healthy children: methodology and discussion. J Clin Sleep Med. 2016;12(11):1549-61.

7. Llaguno NS. Atividade, repouso e cognição de crianças escolares [dissertação]. São Paulo: Universidade Federal de São Paulo; 2020.

8. Hale L, Guan S. Screen time and sleep among school-aged children and adolescents: a systematic literature review. Sleep Med Rev. 2015;21:50-8. 
9. Carter B, Rees P, Hale L, Bhattacharjee D, Paradkar MS. Association between portable screen-based media device access or use and sleep outcomes: a systematic review and meta-analysis. JAMA Pediatr. $2016 ; 170(12): 1202-8$.

10. Irish LA, Kline CE, Gunn HE, Buysse DJ, Hall MH. The role of sleep hygiene in promoting public health: A review of empirical evidence. Sleep Med Rev. 2015;22:23-36.

11. Santos IS, Bassani DG, Matijasevich A, Halal CS, Del-Ponte B, da Cruz $\mathrm{SH}$, et al. Infant sleep hygiene counseling (sleep trial): protocol of a randomized controlled trial. BMC Psychiatry. 2016;16(1):307.

12. Gathecha E, Rios R, Buenaver LF, Landis R, Howell E, Wright S. Pilot study aiming to support sleep quality and duration during hospitalizations. J Hosp Med. 2016;11(7):467-72.

13. Doi L, Wason D, Malden S, Jepson R. Supporting the health and wellbeing of school-aged children through a school nurse programme: a realist evaluation. BMC Health Serv Res. 2018;18(1):664.

14. Sousa CS, Turrini RN. Validação de constructo de tecnologia educativa para pacientes mediante aplicação da técnica Delphi. Acta Paul Enferm. 2012;25(6):990-6.

15. Polit DF, Beck CT. Fundamentos de pesquisa em enfermagem: avaliação de evidências para a prática da enfermagem. 7a ed. Porto Alegre: Art Med; 2011.

16. Associação Portuguesa de Sono; Sociedade Portuguesa de Pediatria. Higiene do sono da criança e do adolescente - ter um bom sono é um sonho possível! Lisboa: Associação Portuguesa do Sono; Sociedade Portuguesa de Pediatria; sd. [citada 2020 Fev 4]. Disponível em: http:// criancaefamilia.spp.pt/media/124389/HIGIENE\%20S0N0\%20 CRIANcA\%20ADOLESCENTE.pdf

17. Associação Brasileira do Sono. Cartilha do sono. Respeite seu sono e siga seu ritmo. Semana do Sono; 2018 Mar 11-18; São Paulo. São Paulo: Associação Brasileira do Sono; 2018 [citada 2020 Fev 4]. Disponível em: http://semanadosono.com.br/assets/cartilha_do_sono_2018.pdf

18. Coluci MZ, Alexandre NM, Milani D. Construção de instrumentos de medida na área da saúde. Cien Saude Colet. 2015;20(3):925-36.

19. Polit DF, Beck CT. The content validity index: are you sure you know what's being reported? Critique and recommendations. Res Nurs Health. 2006;29(5):489-97.

20. Figueiredo RC, Miranda MA, Teles MW, Silva LS, Montalvão AS, Eulálio IS, et al. Educação em saúde escolar e colaboração do enfermeiro: sob a ótica dos educadores da escola. Rev Ceres. 2016;8(1):145-63.

21. Wong ST, Saddki N, Tin-Oo MM. Readability and suitability of oral health education pamphlets produced by the Ministry of Health Malaysia. Med J Malaysia. 2019;74(4):312-9.
22. Mirzaei N, Dehdari T, Taghdisi MH, Zare N. Development of an instrument based on the theory of planned behavior variables to measure factors influencing Iranian adults' intention to quit waterpipe tobacco smoking. Psychol Res Behav Manag. 2019;12:901-912.

23. Grillo RM, Prodócimo E. Jogo e educação: as ludotecas. Educar Rev. 2016;59:299-304

24. Okuhara T, Ishikawa H, Okada M, Kato M, Kiuchi T. Designing persuasive health materials using processing fluency: a literature review. BMC Res Notes. $2017 ; 10(1): 198$.

25. Arora C, Sinha B, Malhotra A, Ranjan P. Development and Validation of Health Education Tools and Evaluation Questionnaires for Improving Patient Care in Lifestyle Related Diseases. J Clin Diagn Res. 2017;11(5):JE06-09.

26. Reina-Bueno M, González-López JR, López-López D, Calvo-Lobo C, Ballesteros-Mora M, Rodríguez-Moreno I, et al. Development and validation of the overall foot health questionnaire for patients with rheumatoid arthritis: a cross-sectional descriptive analysis. Medicina (Kaunas). 2019;55(6):290.

27. Galdino YL, Moreira TM, Marques AD, Silva FA. Validação de cartilha sobre autocuidado com pés de pessoas com diabetes mellitus. Rev Bras Enferm. 2019;72(2):780-7.

28. Santos TC, Martino MM, Sonati JG, Faria AL, Nascimento EF. Qualidade do sono e cronotipo de estudantes de enfermagem. Acta Paul Enferm. 2016;29(6):658-63.

29. Hilt R. Sleep Hygiene for Children [Internet]. In: Patient and Family Education. Seattle (WA): Seattle Children's; 2017 [cited 2020 Feb 4]. 2 p. Available from: https://www.seattlechildrens.org/globalassets/ documents/healthcare-professionals/pal/family/family-sleep-hygiene. pdf

30. American Thoracic Society. Healthy sleep in children. J Respir Crit Care Med. 2017;195:11-2. [cited 2020 Nov 20]. Available from: https:// www.thoracic.org/patients/patient-resources/healthy-sleep-inchildren.pdf

31. Sociedade Brasileira de Pediatria. Higiene do Sono. 2017. 9p. [citado 2020 Dez 3]. Disponível em : https://www.sbp.com.br/fileadmin/user_ upload/19807c-DocCient_-_Higiene_do_Sono.pdf

32. Halal CS, Nunes ML. Education in children's sleep hygiene: which approaches are effective? A systematic review. J Pediatr (Rio J). 2014;90(5):449-56.

33. Bröder J, Okan O, Bauer U, Bruland D, Schlupp S, Bollweg TM, et al. Health literacy in childhood and youth: a systematic review of definitions and models. BMC Public Health. 2017;17(1):361. 